Median number of drugs for each patient was 4 and a number of drugs were implicated to cause hyponatremia.55\% of patients were found to have a diagnosis of SIADH,the other causes were extrarenal solute loss(18\%), Pseuohyponatremia $(18 \%)$ and cirrhosis (9\%). Only 45\% were treated with Tolvaptan,while the rest had sodium replacement, fluid restriction and other supportive care. $27 \%$ died during the admission and $36 \%$ died within the next 6 months of discharge.

Conclusions SIADH is not always the cause of hyponatremia in palliative care patients, hence there is a need for accurate diagnosis of patients to enable a targeted management strategy.

\section{THE ROLE OF PALLIATIVE MEDICINE IN END STAGE DUCHENNE MUSCULAR DYSTROPHY}

Brian Mburu, Derek Willis. Severn Hospice

\subsection{6/bmispcare-2019-ASP.178}

Background Duchenne Muscular Dystrophy (DMD) is a progressive muscle wasting disease. It is the most common type of muscular dystrophy in the UK. Those affected usually die in their late $20 \mathrm{~s}$ or early $30 \mathrm{~s}$ due to cardiac or respiratory problems. As the disease progresses they may need management of symptoms arising from complications of late disease, such as pain. This poster looks into the joint services provided by the community palliative team at Shrewsbury and Telford Severn hospice and the Oswestry neuromuscular clinic caring for five patients with advanced Duchenne muscular dystrophy.

Methods The notes from the clinic consultations of the five patients involved were perused and the consultations noted, along with the proposed management plan. Subsequent clinic follow ups were looked into to review response to the management plan and note any amendments to treatment that were made.

Results The five patients presented with pain as their main symptom. Different choices of analgesia were tried with different responses. All patients reported improvement in their symptoms after starting analgesia with some requiring a combination of analgesics. In addition to this, some of the patients required input from other services regarding other aspects of patient care, such as the respiratory team for sleep studies and assessment for ventilatory support, or access to the hospice for various social events in a safe and welcoming environment.

Conclusion As there are no guidelines for management of DMD, management may involve trials of different analgesia for pain management in addition to involvement of a multidisciplinary team to address other care issues. As palliative medicine involves symptomatic treatment of patients with advanced and terminal disease and holistic care, there is a role for palliative medicine in management of patients with end stage Duchenne muscular dystrophy.

\section{CANNABIS USE IN A RURAL PALLIATIVE CARE COMMUNITY: A SURVEY}

Lynn Davies, Alice O'Connor, Caroline Hart. Severn Hospices, Shropshire

10.1136/bmjspcare-2019-ASP.179

Interest in Cannabis as a medicinal product is growing globally; recent scheduling review of Cannabis by the Chief
Medical Officer puts forward a strong case for the therapeutic benefit of cannabis-based medicinal products. We have noticed an increased incidence of questions, from patients and carers relating to Cannabis use. More patients appear to confide in us about their use of Cannabis. The incidence of Cannabis use in a palliative care population is little known.

We undertook a sequential survey of fifty patients referred into and triaged as requiring domiciliary review by the Severn Hospice Nurse Outreach team. Each patient was asked at the end of the usual initial assessment whether they would take part in the anonymised survey.

Results 50 consecutive patients referred to the Shropshire community palliative care team (outreach specialist nurses) 36 patients completed the survey.

Of 36 questionnaires completed 8 patients declined completion. 10 patients (almost a third of those completing the survey) had considered using Cannabis, 18 had not.

The study population $(n=36)$ were mainly aged over 70 $(n=21)$. Gender split was fairly equal. Of those who had considered cannabis $(n=10)$, the main reason was for pain relief $(n=6)$. Other reasons included general wellbeing and treatment/cure. Half of those who had considered cannabis were on chemotherapy. Ages in the 'cannabis curious' group tended to be younger - with $40 \%$ aged less than 70 . Half of these had researched Cannabis using only Media sources.

Conclusions In a survey of 50 palliative care patients in a rural county of England asked 'Have you considered the use of Cannabis' most had not. Of 10 patients who said they had considered it, the most commonly cited reason was Pain relief.

\section{SYMPTOM CONTROL OF DYSPNOEA IN PATIENTS WITH CYSTIC FIBROSIS}

Claribel Simmons, Krishna Dani. Health Education North East, NHS Greater Glasgow and Clyde

10.1136/bmjspcare-2019-ASP.180

Background Cystic fibrosis (CF) remains an incurable condition in respiratory medicine. One of the main symptoms is dyspnoea. CF is a multi-system disorder and treatment of pulmonary symptoms tends to focus on medication which can be nebulised therefore minimizing the systemic side effects. Traditionally in palliative medicine opioids are effective at relieving dyspnoea however nebulised opioid is not routinely recommended. In patients with CF who are at risk of gastrointestinal complications such a distal intestinal obstruction syndrome (DIOS), administration of nebulised opioids has been sporadically reported to improve dyspnoea and minimize the risk of constipation.

The aim of this systematic review was to examine the role of nebulised opioids in improving dyspnoea in patients with CF.

Methods Medline (1946-2018) and Embase (1974-2018) were searched. Eligible studies met the following criteria: patients with $\mathrm{CF}$, patients $>13$ years of age

Results 70 studies were eligible. Three studies fulfilled the criteria for review. The three studies described different case reports of nebulised opioid in patients with CF. The ages ranged from 13 years to 48 years. All patients had end stage respiratory failure due to CF. Two patients received nebulised morphine and one patient received nebulised fentanyl. 
Improvements in dyspnoea were graded according to improvement in BORG score. An adverse effect of a headache was noted in one patient occurring 48 hours after nebulised morphine had commenced.

Conclusion Nebulised morphine and fentanyl have been used to improve dyspnoea due to $\mathrm{CF}$ in a very limited number of patients, therefore no conclusion advocating the use of nebulised opioids can be made. Further research in the form of randomised controlled trials is needed to improve the evidence base of symptom control in patients with CF. This research must also focus on adverse gastrointestinal side effects such as constipation increasing the risk of DIOS.

\section{RECOGNITION OF SEPSIS IN HOSPICE PATIENTS WITH FEVER}

Rachel Whitehorn. Douglas Macmillan Hospice

\subsection{6/bmjspcare-2019-ASP.181}

Background Historically, patients in hospices have not had routine physiological observations recorded, with the focus being on maintaining comfort at end of life. In recent years, more patients admitted to hospices still wish to receive active life-prolonging treatment, including for sepsis. NICE issued guidance on the recognition of possible sepsis in the community and hospital settings in summer 2016. Recognition includes assessment of patients' basic physiological observations.

This audit was performed to assess the extent to which basic physiological observations are completed to allow careful assessment of patients in the hospice for the presence of sepsis.

Assessment for sepsis risk includes

- Presence of risk factors

- altered conscious state,

- reduction in functional ability

- respiratory rate

- new need for oxygen to maintain saturations

- heart rate, blood pressure

- skin/lip/tongue colour

- temperature

- when the patient last passed urine

- Site of any likely infection.

Method retrospective review of patient records.

Results 15 episodes of pyrexia were identified in 11 patients in a four month period. Two of these were related to blood transfusion and not included in analysis. On assessment of the patients, blood pressure, heart rate, conscious state and functional level were the most frequently recorded sepsis risk factors. Skin colour, respiratory rate, presence of signs of skin or wound infection and urine output were hardly ever or never recorded as assessed.

Nursing staff requested medical assessment in all cases although this could be delayed until normal working hours. Intravenous antibiotics were started in two patients and oral antibiotics given in five. Ceiling of care was discussed in three cases.

Conclusion Decisions are made about the presence of sepsis and the use of antibiotics based on a limited patient assessment. Staff need to be reminded that fuller assessment of basic physiological observations may improve decisionmaking.

\section{OPIOID AUDIT - IN PURSUIT OF APPROPRIATE SYMPTOM MANAGEMENT}

Elizabeth Heard, Alison Franks. University Hospitals Coventry and Warwickshire NHS Trust

\subsection{6/bmjspcare-2019-ASP.182}

Background In a recent spate of referrals regarding pain control we found constipation was the major issue. This prompted us to question the commonality of this, as assessing and managing pain are core medical skills which, if done inadequately, can result in incorrect/inappropriate patient management.

Opioids, an important part of pain management, are widely used, by all doctors. The common side effects are well recognised. There are several different opioids, all of which can cause temporary nausea/vomiting and persistent issues with constipation. Prescribing errors are not uncommon. Thus we conducted an in-patient, Trust-wide spot audit of drug charts for prevalence of opioid prescribing, and anticipation of common side effects.

Method All adult care (non-ITU) wards were visited, drug charts reviewed and spreadsheet data collection tool populated.

Results In total, 782 charts were assessed - 440 (56\%) included an opioid.

Morphine was commonest, 55\%, codeine 32\%, oxycodone $4 \%$, fentanyl 3\%, buprenorphine, methadone and tramadol $2 \%$ each, dihydrocodeine $1 \%$, one prescription each for pethidine and alfentanil.

Of the prescriptions, 229 were for 'regular' opioids and 418 'as needed' - 28 (4\%) of these did not specify frequency of opioid use.

Bowel habit was recorded on $69 \%$ of the charts. At least one laxative was prescribed on 267 charts (61\%), totalling 450 prescriptions - senna 176 prescriptions, lactulose 144, macrogol 77, docusate 31 , glycerine suppositories 11 , six phosphate enemas and one for naloxegol.

Conclusions Appropriately used, opioids are invaluable for managing pain, but cause well recognised common side effects of which constipation can cause the greatest pain, distress and misery for our patients. It's relatively straightforward to manage, but needs to be anticipated, monitored for and addressed effectively to minimise patients suffering because we aren't questioning and recognising symptoms for what they are and prescribing correctly. More education planned.

\section{SURPRISING FINDINGS IN TRACKING PAIN PROGRESSION IN AN INPATIENT COHORT WHO WERE ADMITTED TO A SPECIALIST INPATIENT UNIT}

Abigail Coutinho, Rachel Whitehorn, Emer McKenna. Douglas Macmillan Hospice

10.1136/bmispcare-2019-ASP.183

We aimed to identify whether we are managing our patients pain symptoms effectively using the IPOS as a measuring tool. IPOS forms part of the validated OACC suite of outcomes measures. And also to validate our approach to pain management across all categories and severity of pain and address any deficiencies found. We studied patients over a 3 month period. Interestingly, in the patients with starting higher pain scores $3-4$, we found that $53 \%$ of patients showed improvements in their pain scores, 38\% did not show any 